\title{
Role of MB Isoforms (Isomers) in the Early Diagnosis of Acute Myocardial Infarction
}

\author{
Ranjeet Baral, Xueru Luo, Hitoshi Watanabe*, Ikuhiro Yamasawa and Chiharu IbukiYama \\ In addition to electrocardiogram (ECG), analysis of creatinine phosphokinase (CPK), lactate \\ dehydrogenase (LDH) and their isoenzymes is commonly employed to diagnose acute myocardial \\ infarction (AMI). The aim of this study was to observe the efficiency of isomers of CPK for early \\ diagnosis of AMI. ECG, though useful in many ways, is not $100 \%$ sensitive. The drawback in- \\ volved with CPK and LDH isoenzymes is the time lag factor in diagnosing AMI early enough for \\ interventional therapy. Recently the subunits of these isoenzymes, namely MM and now the MB \\ isomers are being tested for their effectiveness in the early diagnosis of AMI. In our experience MB \\ isomers were found to be accurate markers in the early diagnosis of AMI. They were especially \\ valuable in diagnosing myocardial infarction not detectable by ECG in cases such as non-Q AMI, \\ cardiomyopathy, and bundle branch block among other such cases.
}

(Internal Medicine 33: 210-215, 1994)

Key words: ECG (electrocardiogram), CPK (creatine phosphokinase), LDH(lactate dehydrogenase)

\section{Introduction}

The last decade has been significant progress in the enzymatic diagnosis of acute myocardial infarction (AMI) (1-4). Creatinine phosphokinase (CPK), lactate dehydrogenase (LDH), and their isoenzymes are used in addition to electrocardiogram (ECG) in many hospitals. Recently we have seen the evolution of the subtypes of CPK isoenzymes, namely isomers (isoforms) $(5,6)$ which are subtypes or variants of isoenzymes obtained by high voltage electrophoresis. These CPK isomers, the MM isomers and the cardio-specific marker MB isomers, have proven to be highly efficient in the diagnosis of AMI (7).

Our main research goal was to verify the specificity of CPK$\mathrm{MB}$ isomers as an early, accurate, biochemical marker for the diagnosis of AMI. Early diagnosis is beneficial both in the decision to initiate thrombolytic therapy for salvaging maximum myocardial tissue and for the assessment of reperfusion (8-11). Other conventional markers such as total CPK-MB, $\mathrm{CPK}$ and its isoenzymes are useful but the time lag factor in diagnosing AMI early enough reduces their significance. ECG lacks $100 \%$ specificity or sensitivity in the diagnosis of AMI which is why the isomers are very useful, particularly in cases difficult to diagnose by ECG where no ST-T changes or Q waves are elicited, as well as in cases in which the ECG pattern is atypical, as in bundle branch block and cardiomyopathy. We have been measuring the values of CPK isomer/isoenzymes and LDH isoenzymes in all patients admitted to the emergency department with symptoms of AMI $(12,13)$. In our experience, MB isomers are sensitive indicators for the diagnosis of AMI. One of the drawbacks we faced was the late admission of patients tolerating their initial symptoms of AMI.

\section{Materials and Method}

\section{Patients}

We measured the serial values of CPK isomer/isoenzymes and LDH isoenzymes using a rapid electrophoretic analyzer supplied by Helena Laboratories, Beaumont, Texas, U.S.A. Between September, 1991 and June, 1993 a total of twenty-five patients were admitted to the emergency department with symptoms of AMI in approximately one and half year period. The diagnosis of AMI was confirmed by other investigations including ECG, CAG, and echocardiography. Another twenty subjects were selected as controls. A schematic diagram summarizing the isomer/isoenzyme testing procedure is shown in Fig. 1. The first sample was taken immediately upon admission to the emergency department with complaints of chest pain (discomfort), dyspnea, and other AMI-related symptoms (14, 15). Subsequent serial samples were taken at 2 -hour intervals ( 4 times), 4-hour intervals (4 times), and at 12-hour intervals (2

From the Second Department of Internal Medicine, Tokyo Medical College, Tokyo and *Helena Laboratories, Tokyo

Received for publication August 10, 1993; Accepted for publication February 2, 1994

Reprint requests should be addressed to Dr. Ranjeet Baral, Internal Medicine-Division 2, Tokyo Medical College Hospital, 6-7-1 Nishishinjuku, Shinjuku-ku, Tokyo 160 


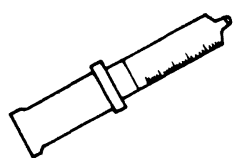

$5 \mathrm{ml}$ blood collected, centrifuged for ten minutes at $3000 \mathrm{rpm}$, serum decanted,

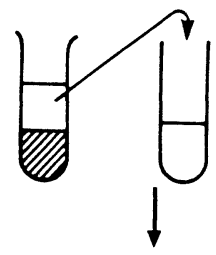

75 ul pipetted into each sample cup.

After reagent addition, gel plate is positioned in the REP unit which automatically performs electrophoresis, then incubates and dries the agarose gel.

$$
\downarrow
$$

The isoform gel is visually inspected under uv light. Finally the densitometer automatically calculates and prints the CPK-MB and CPK-MM values on the computer screen.
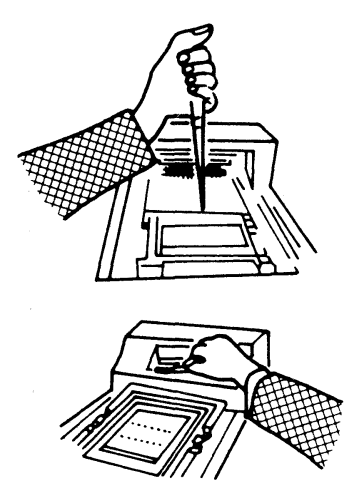

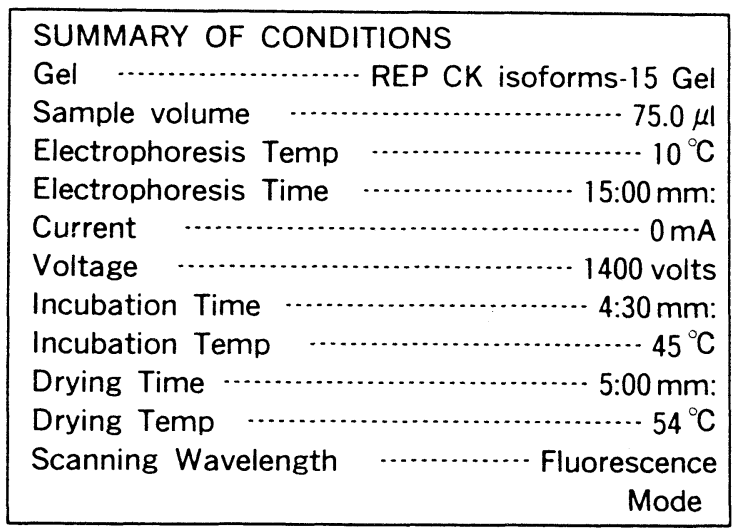

Fig. 1. Schematic illustration of the isomer/isoenzyme testing procedure.

times) for a total of ten samples per patient. The mean values and standard deviation of the MM and $\mathrm{MB}$ isomers taken serially for all the AMI patients are shown in Tables 1 and 2 respectively. Five $\mathrm{ml}$ of blood was centrifuged at $3,000 \mathrm{rpm}$ for 10 minutes. Serum was then removed for immediate assay or stored at $-70^{\circ} \mathrm{C}$. No preservatives or activators were added to the sera. The tests were performed either immediately or within four hours of storage. The samples were subjected to high voltage electrophoresis on agarose gel plates at a low temperature to ensure a rapid separation of the different $\mathrm{CPK} / \mathrm{LDH}$

Table 1. Serial Changes of CPK-MB (\%) Isomers - MB-1, MB-2, Total MB, MB-2/MB-1 Ratio in AMI (Acute Myocardial Infarction) Patients

\begin{tabular}{lrrrrrrrrrr}
\hline MB-1 (Hours) & 2 & 4 & 6 & 8 & 12 & 16 & 20 & 24 & 36 & 48 \\
$\quad$ Mean & 3.27 & 3.82 & 4.05 & 4.15 & 3.81 & 3.72 & 3.20 & 2.85 & 2.17 & 1.87 \\
$\quad$ S.D. & 1.42 & 1.19 & 1.26 & 1.11 & 1.13 & 1.21 & 1.17 & 1.19 & 1.34 & 1.15 \\
\hline MB-2 (Hours) & 2 & 4 & 6 & 8 & 12 & 16 & 20 & 24 & 36 & 48 \\
$\quad$ Mean & 6.60 & 7.44 & 6.66 & 6.05 & 4.50 & 3.27 & 2.12 & 1.63 & 1.23 & 0.99 \\
S.D. & 2.32 & 2.32 & 2.40 & 2.60 & 2.16 & 1.63 & 1.26 & 1.02 & 0.92 & 0.87 \\
\hline MB (Hours) & 2 & 4 & 6 & 8 & 12 & 16 & 20 & 24 & 36 & 48 \\
$\quad$ Mean & 9.77 & 11.26 & 10.71 & 10.20 & 8.32 & 6.99 & 5.31 & 4.48 & 3.39 & 2.86 \\
S.D. & 3.25 & 3.08 & 2.95 & 2.82 & 2.51 & 2.51 & 2.27 & 2.01 & 2.14 & 1.86 \\
\hline (Hours) & & & & & & & & & & \\
MB-2/MB-1 & 2 & 4 & 6 & 8 & 12 & 16 & 20 & 24 & 36 & 48 \\
$\quad$ Mean & 2.30 & 2.04 & 1.76 & 1.55 & 1.27 & 0.90 & 0.64 & 0.57 & 0.55 & 0.50 \\
S.D. & 1.11 & 0.62 & 0.77 & 0.74 & 0.77 & 0.41 & 0.26 & 0.33 & 0.28 & 0.38 \\
\hline
\end{tabular}

S.D.: standard deviation. 
Table 2. Serial Changes of CPK-MM (\%) Isomers-MM-1, MM-2, MM-3, and MM-3/MM-1 Ratio in AMI (Acute Myocardial Infarction) Patients

\begin{tabular}{lrrrrrrrrrr}
\hline MM-1 (Hours) & 2 & 4 & 6 & 8 & 12 & 16 & 20 & 24 & 36 & 48 \\
$\quad$ Mean & 23.17 & 17.99 & 19.62 & 24.66 & 31.40 & 40.91 & 48.08 & 51.17 & 50.97 & 56.31 \\
S.D. & 10.20 & 7.41 & 8.93 & 10.83 & 12.01 & 11.39 & 10.40 & 9.54 & 9.22 & 10.31 \\
\hline MM-2 (Hours) & 2 & 4 & 6 & 8 & 12 & 16 & 20 & 24 & 36 & 48 \\
$\quad$ Mean & 24.05 & 26.70 & 28.85 & 29.63 & 30.06 & 26.94 & 24.59 & 22.92 & 21.73 & 18.54 \\
S.D. & 7.04 & 4.81 & 4.39 & 3.65 & 5.85 & 7.35 & 6.62 & 6.75 & 6.11 & 6.58 \\
\hline MM-3 (Hours) & 2 & 4 & 6 & 8 & 12 & 16 & 20 & 24 & 36 & 48 \\
$\quad$ Mean & 34.89 & 38.57 & 35.05 & 28.64 & 22.00 & 15.83 & 10.63 & 9.71 & 9.15 & 8.44 \\
S.D. & 8.84 & 10.76 & 11.02 & 11.35 & 8.42 & 6.53 & 4.57 & 3.40 & 3.31 & 3.89 \\
\hline (Hours) & & & & & & & & & & \\
MM-3/MM-1 & 2 & 4 & 6 & 8 & 12 & 16 & 20 & 24 & 36 & 48 \\
$\quad$ Mean & 2.05 & 2.61 & 2.52 & 1.59 & 0.85 & 0.46 & 0.26 & 0.20 & 0.19 & 0.16 \\
S.D. & 1.36 & 1.68 & 1.61 & 1.11 & 0.56 & 0.32 & 0.17 & 0.12 & 0.10 & 0.12 \\
\hline
\end{tabular}

S.D.: standard deviation.

bands. The assay is accurate and requires only 30 minutes for the entire process. After electrophoresis the plate was viewed under UV light before being scanned by the densitometer.

\section{Results}

A typical densitometer scanned analysis by the densitometer after electrophoresis of the sera from an AMI case, normal subject, and a non-Q AMI case is shown in Figures 2, 3, and 4, respectively. For the AMI patient, Fig. 2, the MB band is clearly elicited with the MB-2 band (tissue form) taller than the MB-1 band (serum form), the characteristic sequence pattern diagnostic of AMI. MM bands here are also diagnostic of AMI with the tissue form MM-3 band being taller than MM-2 and MM-1 bands $(16,17)$. MM isomers are sensitive markers of AMI but

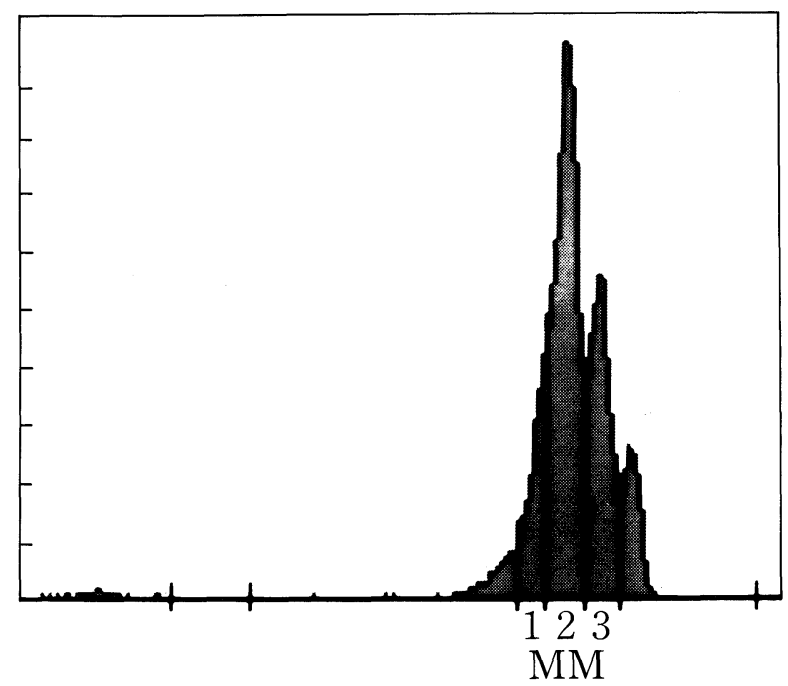

Fig. 3. Electrophoretic analysis of a normal case.

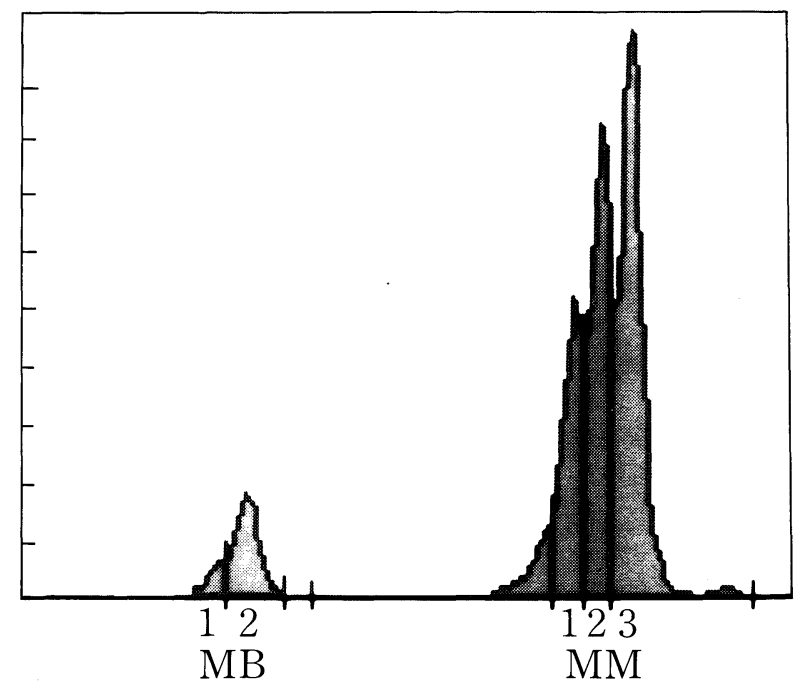

Fig. 2. Electrophoretic analysis of an acute myocardial infarction (AMI) case.

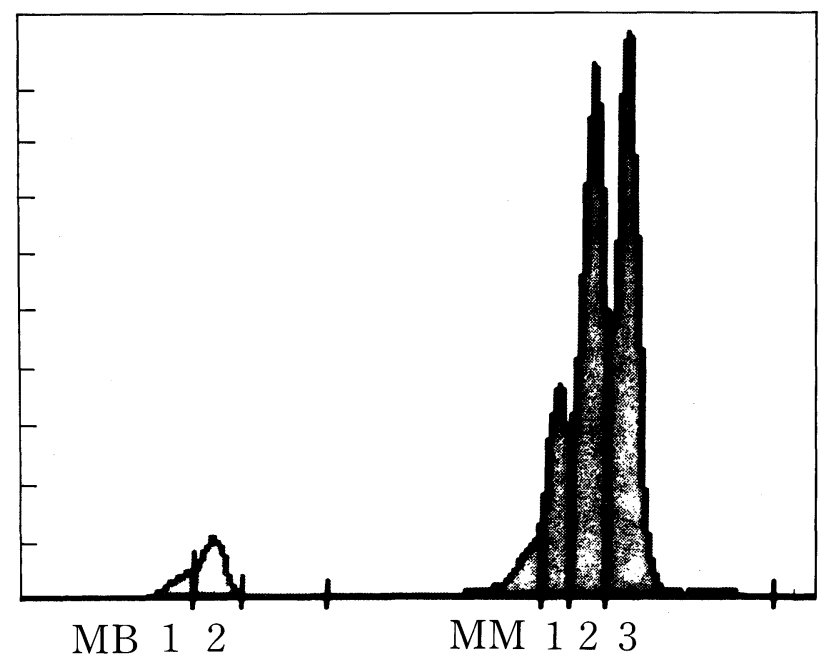

Fig. 4. Electrophoretic analysis of a non-Q wave AMI (acute myocardial infarction) case. 
they are not cardio-specific, since they are present in skeletal muscles as well as other tissues, whereas MB isomers are largely present only in the myocardium. They comprise $40 \%$ of $\mathrm{CPK}$ in the myocardium and are present in insignificant amounts in the rectus abdominus, gastrocnemius, and some other muscles. The pattern of a normal case, as seen in Fig. 3, shows no MB band elicited in the isomer analysis and the serum form, (MM 1 band) is greater than the tissue form bands (MM-2 and MM-3). The isomer pattern of a non-Q AMI patient is identical to that of a typical AMI case, as can be seen in Fig. 4. The histogram in Fig. 5 depicts the isomer values of normal subjects used as controls. There was practically no elicitation of MB isomer in these cases and the MM-1 band (serum form) was of a higher value than the MM-2 and MM-3 bands, i.e. the tissue form bands. This is exactly the reverse of the sequence pattern to that of an AMI case. The graph in Fig. 6 indicates the serial changes of the different MB fractions in AMI patients from the time of admission through the next forty-eight hours. There is an early rise of total $\mathrm{MB}, \mathrm{MB}-2$, and the $\mathrm{MB}-2 / \mathrm{MB}-1$ ratio following AMI and they then decline gradually to normal levels within the next 48 hours. MB isoforms are elicited as early as two hours after chest pain and they peak at 4-8 hours. Similarly, Fig. 7 depicts the serial changes of the different MM fractions. Total MM, MM-3 and MM-3/MM-1 ratios also show early elicitation following AMI. The sample sequences were taken immediately after admission of patients to the emergency department. Thus we must keep in mind that zero hour does not indicate the time of occurrence of AMI (patients tend to tolerate their initial symptoms) but rather the first sample after admission. However, the usefulness of isomers is clear, especially in cases difficult to diagnose by ECG.

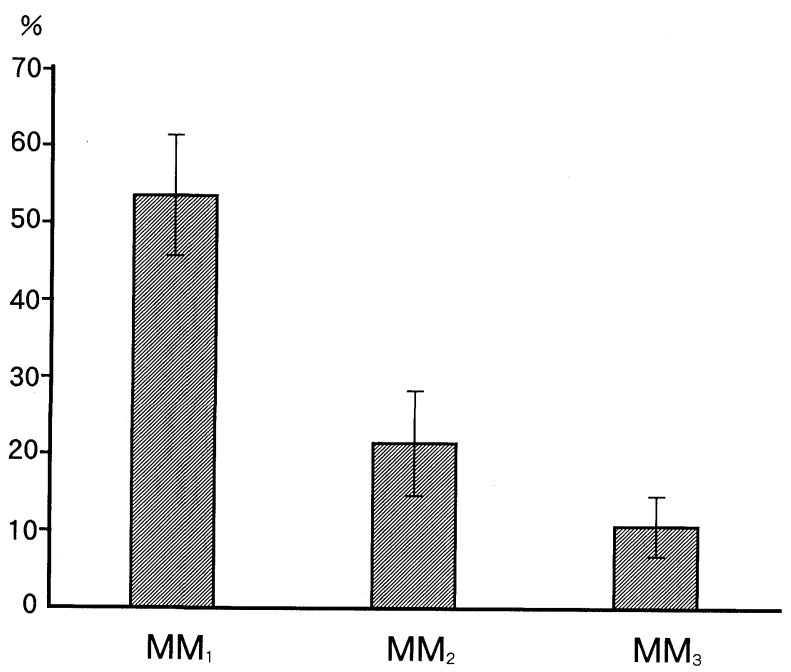

Fig. 5. Control evaluation of creatinine phosphokinase (CPK) isomer values in normal subjects.
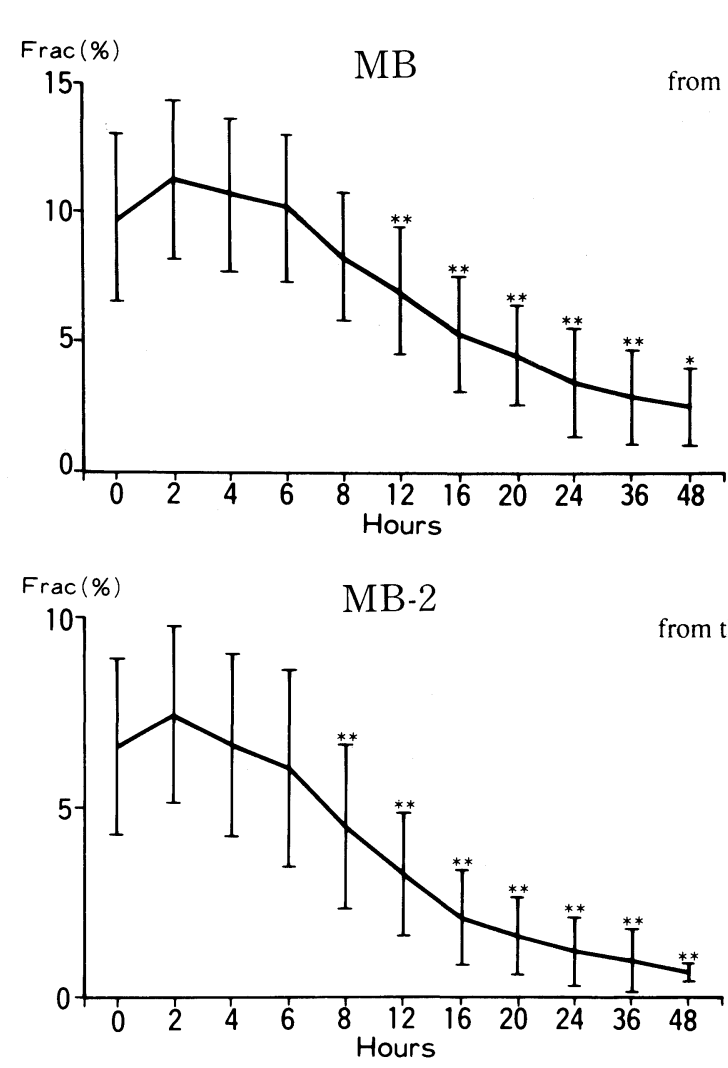
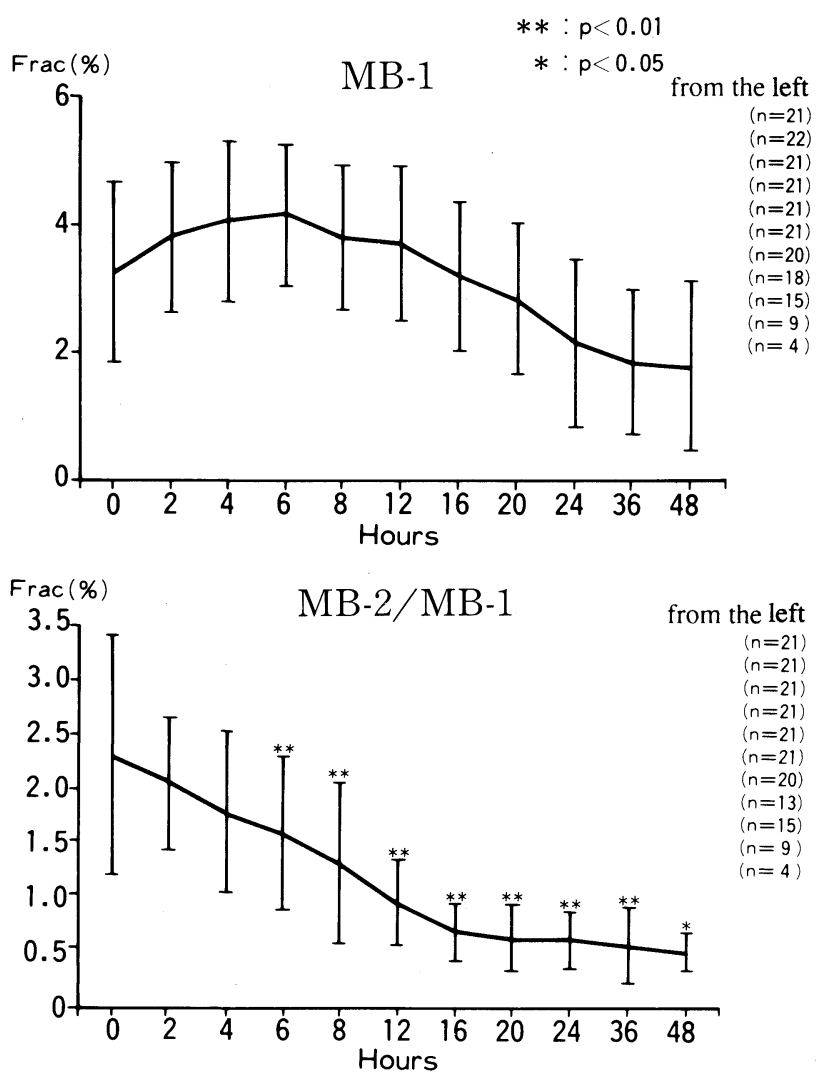

Fig. 6. Graphic representation of the serial changes of (a) total MB isomer, (b) MB-1 isomer, (c) MB-2 isomer, and (d) MB-2/MB-1 isomer ratio in AMI patients. 

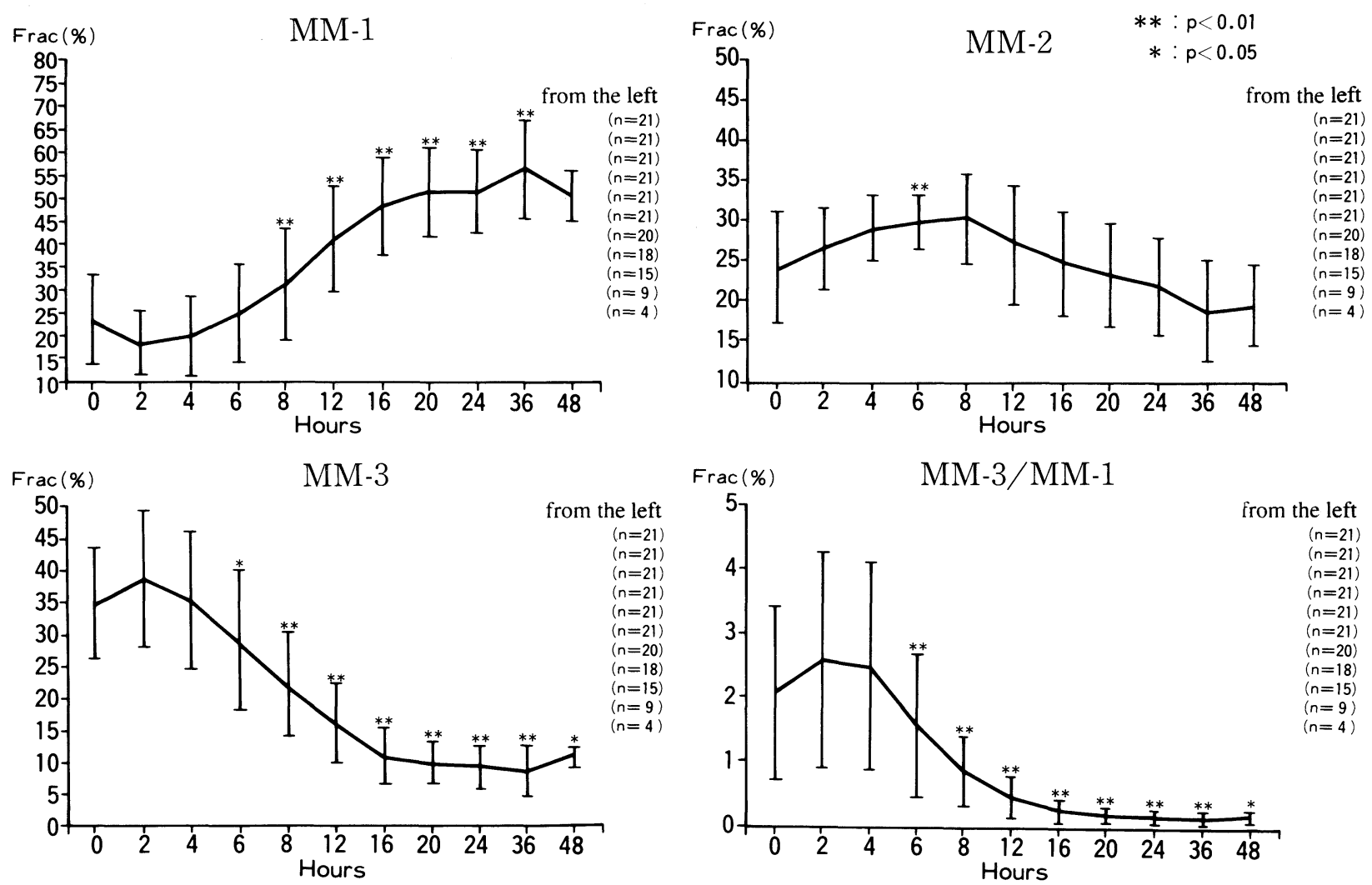

Fig. 7. Graphic representation of the serial changes of (a) total MM-1 isomer, (b) MM-2 isomer, (c) MM-3 isomer, and (d) MM-3/MM-1 isomer ratio in acute myocardial infarction (AMI) patients.

\section{Discussion}

In the present study CPK isomers were diagnostic for all AMI cases. They are a useful asset in the early diagnosis of AMI, especially in cases of doubt. Even though ECG still remains the method of choice for all practical purposes, its sensitivity cannot always be relied upon. Fifty percent of AMI patients initially present to the emergency department with nondiagnostic ECGs, whereas the sensitivity of isomers is 92$100 \%$ accurate as early as 3 hours following AMI (18). In the present study isomers were useful in the diagnosis of non-Q AMI, where the ECG fails to elicit the ST-T changes and later the $\mathrm{Q}$ wave, the diagnostic electrographic pattern of typical AMI. They would also be useful if patients with bundle branch block, cardiomyopathy, and other such cases were to experience myocardial infarction, wherein the diagnosis of AMI would be difficult to determine solely on the basis of ECG.

One of the problems we faced was the late admission of patients following AMI because they tolerate their initial symptoms. Also AMI patients frequently are unable to give a clearcut time of symptom onset. A rare obstacle we came across was regarding macroanomaly cases, which tend to have elevated values of CPK-MB. Although they are not associated with any known pathology, the possibility of misdiagnosis regarding myocardial infarction cases must be borne in mind as the MB isomer is not only elicited in these cases, but the sequence pattern can be identical to that of AMI cases. It has been reported that anti-CPK MB monoclonal antibodies are specific for CPK$\mathrm{MB}$ without cross-activity against MM, BB, or mitochondrial $\mathrm{CPK}$, and therefore are free from interference due to the presence of macro-CPK (19). CPK-MM had been used rather than MB for AMI detection because of the limitations of the assay lacking analytical sensitivity and specificity $(20,21)$. The other possible reason given was that MM peaked earlier than MB because MM is located in the myofibrils and is bound reversibly, whereas MB is located within the cell (cytosolic). But MM, unlike MB, is not specific for the myocardium and is present in other muscular tissue. Thus the CPK-MM sequence pattern after prolonged exercise in normal subjects is similar to that of an AMI case but the MB isomer pattern is normal. Concerning the CPK of the human myocardium, $25-40 \%$ of it occurs as MB-2, the tissue form (gene product). The carboxypeptidase hydrolysis of C-terminal lysine residues produces the post-translational product, CPK-MB-1 (22).

The role of MB isomers is also vital in the assessment of the success of reperfusion and also in ascertaining if thrombolytic treatment is necessary (23-27). Thrombolysis in the absence of AMI possesses a $0.5-1 \%$ risk of hemorrhage and can even lead to death. The most effective time period for using thrombolytic agents is within 4-6 hours of AMI. Thus, the CPK-MB isoform 
would be ideal for this kind of therapeutic purpose also.

Since isoforms are more specific and sensitive and they can be detected earlier than the isoenzymes or total CPK it may have a future role in cardiac diseases other than myocardial infarction $(28,29)$. Previous research has shown that transient CPK elevations in unstable angina signifies a risk. Such cases have a higher one year mortality rate. At present we are investigating whether isoforms are elicited following reversible ischemic damage to the myocardium. Early diagnosis is important in order to make the decision to initiate thrombolytic therapy which is vital to the successful treatment of the AMI patient. Isoforms are invaluable in cases difficult to diagnose by ECG. We would like to point out that this paper does not intend to highlight the specific chronological sequence of events following AMI but rather the significance of isomers in a clinical setting regarding the diagnosis of AMI. Thus, isoforms (isomers) are likely to play a supportive role to ECG in the diagnosis of AMI and other related cardiac diseases.

Acknowledgments: The authors would like to thank the staff of Helena Laboratories for technical support and assistance in the use of their equipment. We are also grateful to the staff of the Emergency Department and the nursing staff of the Coronary Care Center for their sincere service. Lastly we would like to thank Professor Patrick Barron and his secretary, Miss Chiharu Sakata, for their continuous support as well as reviewing the manuscript more than once.

\section{References}

1) Wagner GS, Roe CR, Limbird LE, et al. The importance of identification of the myocardial-specific isoenzyme of creatine phosphokinase (MB Form) in the diagnosis of acute myocardial infarction. Circulation 47: 263, 1973.

2) Wright LT. Changes in cardiac-enzyme analysis in diagnosis of acute myocardial infarction. Clin Lab Science 1(6): 355, 1988.

3) Roberts R, Gowda KS, Ludbrook PA, et al. Specificity of elevated serum MB creatine phosphokinase activity in the diagnosis of acute myocardial infarction. Am J Cardiol 36: 433, 1975.

4) Irvin RG, Cobb FR, Roe CR. Acute myocardial infarction and MB creatine kinase phosphokinase. Arch Intern Med 140: 329, 1980.

5) Puleo PR, Blick D, Guadgno PA, et al. Early diagnosis of myocardial infarction based on assay for sub-forms of creatine kinase-MB. Circulation 82: 759, 1990.

6) Morelli RL, Carlson CJ, Emilson B, et al. Serum creatine kinase isoenzyme sub-bands after acute myocardial infarction in man. Circulation 67: 1283, 1983.

7) Panteghini M, Cuccia C. Isoforms of creatine kinase $M M$ and $M B$ in acute myocardial infarction. Clin Chem Acta 155: 1, 1986.

8) Apple FS, Sharkey SW, Werdick M, et al. Analysis of creatine kinase isoenzymes and isoforms in serum to detect reperfusion after myocardial infarction. Clin Chem 33(4): 507, 1987.

9) Puleo PR, Perryman MB, Bressner MA, et al. Creatine kinase isoform analysis in the detection and assessment of thrombolysis in man. Circulation 75: 1162, 1987.
10) Roberts R. Reperfusion and the plasma isoforms of creatine kinase isoenzymes: a clinical perspective. J Am Coll Cardiol 9: 464, 1987.

11) Escobar R, Gornet TG, Wu AHB. Evaluation of an automated electrophoresis analyzer for cardiac isoenzymes. Helena Rep. Clin Chem 33: 1284, 1988 (Abstract).

12) Wu AHB, Gornet TG, Bretaudiere JP, et al. Comparison of enzyme immunoassay and immunoprecipitation for creatine kinase $\mathrm{MB}$ in diagnosis of acute myocardial infarction. Clin Chem 31: 470, 1985.

13) Lee TH, Weisburg MC, Cook EF, et al. Creatine kinase and CK-MB in emergency rooms. Arch Intern Med 147: 1151, 1987.

14) Viskin S, Heller K, Gheva D, et al. The importance of CK determination in identifying acute myocardial infarction among patients complaining of chest pain in an emergency room. Cardiology 74: 100, 1987.

15) Apple FS. Creatinine kinase MM isoforms. Clin Chem News 8(2): 8 , 1988.

16) Morelli RL, Emilson B, Rapaport E, et al. MM-CK subtypes diagnose reperfusion early after myocardial infarction. Am J Med Sci 293(3): 139, 1987.

17) Puleo PR, Guadagno $P$, Scheel M, et al. Diagnostic accuracy of a rapid MB-CK subform assay in the early hours of myocardial infarction. Clin Chem 35(6): 1119, 1989.

18) Vaidya HC, Maynard Y, Dietzler DN, et al. Direct measurement of creatine kinase-MB activity in serum after extraction with a monoclonal antibody specific to the MB isoenzyme. Clin Chem 32: 657, 1986.

19) Wu AHB, Gornet TG, Wu VH, et al. Early diagnosis of acute myocardial infarction by rapid analysis of creatinine kinase isoenzyme-3 (CK-MM) sub-types. Clin Chem 33(3): 358, 1987.

20) Hashimoto $H$, Abendschein D, Strauss A, et al. Early detection of myocardial infarction in conscious dogs by analysis of plasma MM creatine kinase isoforms. Circulation 71: 363, 1985.

21) Billadello JJ, Fontanet HL, Strauss AW, et al. Characterization of MB creatine kinase isoform conversion in vitro and in dogs. J Clin Invest 83: 1637, 1989.

22) Puleo PR, Perryman B. Noninvasive detection of reperfusion in acute myocardial infarction based on plasma activity of creatine kinase MB subforms. J Am Coll Cardiol 17(5): 1047, 1991.

23) Christenson RH, Ohman EM, Clemmensen P, Grade P. Characteristics of creatine kinase $\mathrm{MB}$ and $\mathrm{MB}$ isoforms in serum after reperfusion in acute myocardial infarction. Clin Chem 35: 2179, 1989.

24) Garabedian HD, Gold HK, Yasuda T, et al. Detection of coronary artery reperfusion with creatine kinase-MB determinations during thrombolytic therapy: correlation with acute angiography. J Am Coll Cardiol 11: 729, 1988.

25) Panteghini M, Pagani F. Isoforms of creatine kinase isoenzymes in serum in acute myocardial infarction after intracoronary thrombolysis. Clin Chem 33: 2039, 1987.

26) Jaffe AS, Serota H, Grace A, et al. Diagnostic changes in plasma creatine kinase isoforms early after the onset of acute myocardial infarction. Am J Med 85: 353, 1988.

27) Tsukamoto H, Hashimoto H, Matsui $Y$, et al. Detection of myocardial reperfusion by analysis of serum creatine kinase isoforms. Clin Cardiol 11: $287,1988$.

28) Wu AHB. Creatine kinase isoforms in ischemic heart disease. Clin Chem 31: 327, 1987.

29) Lott JA, Stang JM. Serum enzymes and isoenzymes in the diagnosis and differential diagnosis of myocardial ischemia and necrosis. Clin Chem 26: $1241,1980$. 